\title{
SPL8, a local regulator in a subset of gibberellin-mediated developmental processes in Arabidopsis
}

\author{
Yan Zhang $\cdot$ Stefan Schwarz $\cdot$ Heinz Saedler $\cdot$ \\ Peter Huijser
}

Received: 10 July 2006/ Accepted: 13 October 2006

(C) Springer Science+Business Media B.V. 2006

\begin{abstract}
Gibberellins (GAs) are important plant growth regulators, regulating many plant developmental processes, including seed germination, root and stem elongation, rosette expansion, floral induction and anther development. The diverse effects of GAs on plant development make it critical to maintain an appropriate endogenous GA level and a fine-tuned GA signalling. Some global regulators in GA signalling have been identified but little is known about genes specifically involved in local implementation of GA signalling. Here we report that the Arabidopsis thaliana SBP-box gene SQUAMOSA-PROMOTER-BINDING-PROTEIN-LIKE8 (SPL8) acts as a local regulator in a subset of GA-dependent developmental processes. Previous knowledge holds that SPL8 is involved in reproductive development as deduced from its loss-of-function phenotype (Unte et al. (2003) Plant Cell 15:1009-1019). We now determined that constitutive overexpression of SPL8 affects fertility due to non-dehiscent anthers, likely resulting from a constitutive GA response, suggesting a positive role of SPL8 in GA-mediated anther development. On the other hand, SPL8 gain- and lossof-function mutants showed opposite responses to GA and its biosynthetic inhibitor paclobutrazol (PAC) with respect to seed germination and root elongation during
\end{abstract}

Y. Zhang $\cdot$ S. Schwarz $\cdot$ H. Saedler $\cdot$ P. Huijser $(\bowtie)$

Department of Molecular Plant Genetics, Max Planck Institute for Plant Breeding Research, Carl-von-Linné-Weg 10, 50829 Cologne, Germany

e-mail: huijser@mpiz-koeln.mpg.de

Present Address:

Y. Zhang

Plant Gene Expression Center, UC Berkeley, 800 Buchanan

St., Albany, CA 94710, USA the seedling stage. Genes involved in GA biosynthesis and signalling are transcriptionally affected by altered SPL8 expression. Our study uncovered a tissue-dependent regulatory role for $S P L 8$ in the response to GA signalling in plant development.

Keywords Anther development - Arabidopsis . Fertility · Gibberellic acid signalling - SBP-box gene
Abbreviations
GA Gibberellic acid
PAC Paclobutrazol
JA Jasmonate
MeJA Methyl jasmonate
DAG Days after germination

\section{Introduction}

The phytohormone gibberellic acid and its derivatives (GAs) are important plant growth factors regulating many developmental processes, including seed germination, rosette leaf expansion, stem and root elongation, floral induction and anther development (Fleet and Sun 2005). The diverse and vital effects of GAs on plant development require appropriately controlled endogenous GA levels and also fine-tuned GA signal responses. Accordingly, extensive studies have been conducted, mainly through genetic screens, to get a better understanding of the GA biosynthetic and signalling pathways (Olszewski et al. 2002).

In recent years, the GA biosynthetic pathway has become well understood and GA-biosynthetic genes 
have been cloned and characterized from many plant species (Hedden and Phillips 2000). Mutants defective in these genes, like gal-3, show germination failure, dwarfism, late flowering and male sterility. The phenotypic alterations of GA-deficient mutants can be rescued by exogenous application of GAs (Koornneef and Van der Veen 1980).

Further efforts dedicated to comprehend GA signalling gave rise to a generally accepted derepressiblerepression model (Peng et al. 1997). GA-signalling mutants supporting this model can be divided into two major classes according to their characteristic phenotypes. The first class consists of GA-insensitive mutants. They phenocopy GA-deficient mutants but cannot be rescued by exogenous GAs. These mutants carry either dominant mutations in negative regulators or recessive mutations in positive regulators of the GA signalling cascade. For instance, the Arabidopsis $g a$ insensitive (gai) mutant allele conferred dominant GAinsensitivity due to a 17 amino acid deletion within GAI, a DELLA protein negatively regulating GAmediated development (Peng et al. 1997). The deletion of the DELLA domain of the gai allele rendered GAI resistance to GA-dependent derepressing effects (Dill et al. 2004; Fu et al. 2004). The group of recessive GAinsensitive mutants in Arabidopsis is represented by sleepy1 (sly1; Steber et al. 1998). SLY1 encodes an F-box protein involved in GA-dependent DELLA protein degradation (McGinnis et al. 2003; Dill et al. 2004).

The second major class of GA signalling mutants represents recessive mutations in negative regulators of GA signalling, which therefore show a constitutive response to GAs. Constitutive GA response mutants show PAC-resistant seed germination, have pale-green leaves, elongated stems and are male sterile. The Arabidopsis spindly (spy) mutants belong to this group (Jacobsen and Olszewski 1993). SPY encodes a ser/thr $O$-linked $N$-acetylglucosamine transferase and has been proposed to function by competing for protein substrates with phosphatases and/or kinases (Filardo and Swain 2003).

Although quite a few genes have been identified and the derepressible-repression model feasibly proposed, GA signalling on the whole is still elusive. Most GA signalling components characterized so far simultaneously cover a broad range of GA-regulated developmental processes, thus representing global GA response regulators. However, bioactive GAs can have dramatically different effects on plant development depending on tissue type and developmental stage (Fleet and Sun 2005). It is hence reasonable to hypothesize that plants have evolved local acting regulatory networks to maintain appropriate GA levels and signalling in specific tissues and/or developmental stages. Such local regulators have been identified in GA biosynthesis/signalling, albeit few. The Arabidopsis homeobox protein SHOOTMERISTEMLESS inhibits a GA 20oxidase within the shoot apical meristem to ensure a low GA level favourable for maintenance of the meristem (Hay et al. 2002). An AP2-type transcription factor, DWARF AND DELAYED-FLOWERING1 (DDF1), regulates GA biosynthesis in Arabidopsis during stem elongation and floral induction as deduced from its dominant mutant phenotype and expression pattern (Magome et al. 2004). Also Arabidopsis SHORT INTERNODES (SHI) has been proposed to be a local GA-signalling regulator as overexpression of SHI caused mainly shortening of the inflorescence stem and a delay of floral induction (Fridborg et al. 1999).

Here we report that the Arabidopsis SBP-domain protein SPL8 (Cardon et al. 1999) may represent such a local acting regulator in GA signalling. Most likely being a transcription factor (Birkenbihl et al. 2005), SPL8 has previously been shown to be required for proper micro- and megasporogenesis as deduced from its semi-sterile mutant phenotype (Unte et al. 2003). In this study, we generated SPL8 gain-of-function transgenic lines. The phenotypic comparison of SPL8 gain- and loss-of-function suggested that SPL8 affects GA signalling in a tissuespecific way. Our further experimental data provided evidence to support the hypothesis that SPL8 acts locally in a subset of GA-mediated developmental processes.

\section{Material and methods}

Plant material and growth conditions

All mutants and transgenic plants described here were derived from Arabidopsis thaliana ecotype Columbia-0 (wild type). Seeds of the spy-3 mutant were obtained from the Arabidopsis stock centre (NASC). Seeds were stratified for 4 days at $4^{\circ} \mathrm{C}$ before sowing on soil containing a mixture of substrate and vermiculite $(3: 1)$. Seeds for growing on germination media, were surfacesterilized in $98 \%$ ethanol followed by $10 \%$ sodium hypochlorite, and rinsed with sterile water. Plated seeds were kept at $4^{\circ} \mathrm{C}$ for 4 days, then moved to a growth chamber. Plants were grown under controlled environmental conditions as described by Unte et al. (2003). 


\section{Generation of transgenic plants}

For complementation of the spl8 mutant, a 4,588 bp fragment covering the entire SPL8 genomic region was derived from BAC T7I23 through a partial digest with the restriction enzymes BstZ171 and SpeI. After Klenow-filling the ends the fragment was cloned into the SmaI site of the binary vector pGJ2148. To generate the 35S::SPL8 construct, the entire SPL8 coding sequence was subcloned into binary vector pGJ2171 which carries two copies of the Cauliflower Mosaic Virus 35S promoter. Constructs were introduced into plants using Agrobacterium tumefaciens strain pGV3101 (pMP90) by the floral-dip method (Clough and Bent 1998). Transgenic lines were selected on soil by spraying seedlings with $0.1 \%(\mathrm{v} / \mathrm{v})$ BASTA.

\section{Semiquantitative RT-PCR}

Total RNA was extracted using the RNeasy ${ }^{\mathrm{TM}}$ kit together with the On-Column DNase system (Qiagen) according to the manufacturer's instructions. Reverse transcriptase-mediated polymerase chain reactions (RT-PCR) were performed with the Superscript II kit (Invitrogen) in the presence of $\alpha_{-}{ }^{32} \mathrm{PdCTP}$. Samples were normalized based on the radioactivity incorporated as determined by liquid scintillation counting. PCRs were performed under the following three-step cycling conditions: $24-32$ PCR cycles at $94^{\circ} \mathrm{C}$ for $40 \mathrm{~s}$, $60^{\circ} \mathrm{C}$ for $40 \mathrm{~s}$, and $72^{\circ} \mathrm{C}$ for $50 \mathrm{~s}$. All reactions used $1 \mu \mathrm{l}$ cDNA as template in $25 \mu \mathrm{l}$ total volume. After amplification, RT-PCR products were separated from the primers by electrophoresis in $1.2 \%$ agarose gels and quantified by phosphor imaging using ImageQuant software (Molecular Dynamics). Oligonucleotides for the detection of SPL8 (ZY67 ATGTTGGACTACGAATGGGATAATC; ZY66 TTAGGTGGCGGAGGCTGATTGGTG), GA5 (ZY78 CGGTTTCTTC CTCGTGGTCAATC; ZY79 TGATGTGATGCTGTCCAAAAGC), were synthesized by Invitrogen. Primer sequences for RAN3 were as described by Unte et al. (2003), those for $\gamma$-TIP were described by Cowling et al. (1998).

Anther histology, pollen viability and microscopy

Determination of anther histology was performed as described by Sorensen et al. (2002). All light and scanning electron microscopy were performed according to Unte et al. (2003). Pollen viability was determined by FDA-staining (Regan and Moffatt 1990) followed by fluorescence microscopy using the filter combination BP485 (excitation), FT510 (beamsplitter) and LP520 (barrier).

Germination and root length measurement

Sterilized seeds were spread on germination medium (GM; Murashige and Skoog medium, Duchefa) supplemented (where appropriate) with $\mathrm{GA}_{3}$ (Sigma) or PAC (Duchefa) and kept 4 days at $4^{\circ} \mathrm{C}$ for stratification. Because seed storage time has a great impact on germination potential, the experiments were performed using seeds with a similar storage time from all genotypes. Germination was recorded 7 days after the plates had been moved to a growth chamber. The percentage of germination and standard deviation for each genotype were determined from six trials with 60 100 seeds per trial.

To measure root length, seedlings were grown on vertically orientated GM plates supplemented with $\mathrm{GA}_{3}$ or PAC. Seven days after germination (DAG) the seedlings were imaged on a digital flatbed scanner. Root length was determined from the digital images using the Java based image processing program ImageJ (http://rsb.info.nih.gov/ij/).

Hormone and inhibitor treatment

For GA treatment of soil-grown plants, $100 \mu \mathrm{M} \mathrm{GA}_{3}$ (Sigma) in $0.02 \%$ Tween-20 was sprayed twice per week starting with 2-week-old seedlings. For GA or PAC treatment of flowers and seedlings, $100 \mu \mathrm{M} \mathrm{GA}_{3}$ or $10 \mu \mathrm{M}$ PAC in $0.02 \%$ Tween-20 were sprayed. Spraying $0.02 \%$ Tween- 20 was always used as a mock treatment. Plant materials were collected $2 \mathrm{~h}$ after treatment for RNA extraction. For germination assays and root elongation measurements, $10 \mathrm{mM} \mathrm{GA}_{3}$ or PAC stock solutions (in $96 \%$ ethanol) were added to autoclaved GM to their required final concentrations. GM supplemented with a comparable diluted (1:200 to $1: 1000)$ amount of ethanol was used for the nontreated controls. $100 \mu \mathrm{M}$ MeJA (Sigma, USA) were used to treat mature anthers of $35 S:: S P L 8$ transgenic plants for the dehiscence assay as described by Zhu et al. (2004).

Upon request, all novel materials described in this publication will be made available in a timely manner for non-commercial research purposes, subject to the requisite permission from any third-party owners of all or parts of the material. Obtaining any permissions will be the responsibility of the requestor. 


\section{Results}

The spatiotemporal expression of SPL8 is important for plant development

As previously reported by Unte et al. (2003), three spl8 mutants were identified by their semi-sterile phenotype, the severity of which decreased acropetally within the inflorescence. In addition to defects in micro- and megasporogenesis, spl8 mutant flowers were also found to display shorter stamen filaments and a lower trichome density on their sepals, all traits known to be regulated by GAs (Perazza et al. 1998; Cheng et al. 2004). A possible role of SPL8 in GA biosynthesis/ signalling has been suggested (Unte et al. 2003).

In order to investigate this possibility, bioactive GAs were exogenously applied to spl8 mutants but the semisterile mutant phenotype could not be rescued. Therefore, SPL8 is unlikely to be directly involved in GA biosynthesis. To obtain additional clues on the possible role of SPL8 in GA-regulated development, the SPL8 coding sequence under the control of the strong and constitutive $35 \mathrm{~S}-\mathrm{CaMV}$ promoter was introduced into wild type and spl8-1 mutants. Proper transgene expression in the transgenic lines obtained was confirmed by RT-PCR (data not shown).

In the spl8-1 mutant background, the constitutively expressed SPL8 transgene could not restore fertility (Fig. 1A). In fact, all of the six transgenic lines displayed an unexpected enhanced sterility, as also observed upon constitutive over-expression of $S P L 8$ in a wild-type background. The SPL8 gain-of-function lines bear $10-60 \%$ fertile, elongated siliques in their fully developed primary inflorescences, whereas spl8-1 mutant primary inflorescences produce $45-80 \%$ fertile siliques. In addition to sterility, the SPL8 transgenic lines with either a spl8-1 mutant or a wild-type background displayed a consistent and heritable pleiotropic abnormal phenotype. In particular the transgenics showed dwarfism, small and dark-green rosette leaves and a compact inflorescence (Fig. 1A). Furthermore, seed germination rate and root elongation were found to be reduced as discussed below. In wild-type plants, $S P L 8$ is predominantly expressed in inflorescence and siliques, detectable in seedlings and almost undetectable in other vegetative tissues (Fig. 1B). Therefore, we consider the pleiotropic phenotype mostly a result of ectopic expression of SPL8 in tissues where it is normally inactive.

In contrast to this dramatic gain-of-function phenotype, the sterility of $s p l 8$ mutants was fully rescued when spl8-1 and spl8-3 were transformed with SPL8 under the control of its endogenous promoter, as part of a $4.8 \mathrm{~kb}$ genomic fragment spanning the SPL8 genomic locus. These complementation transgenic lines also displayed no discernable morphological defects (data not shown). These results strongly suggest that the spatiotemporal expression of SPL8 is important for proper plant development.

Constitutive SPL8 expression resulted in anther non-dehiscence

It is remarkable that both SPL8 loss- as well as gainof-function resulted in partial sterility. Unte et al. (2003) showed that SPL8 loss-of-function affected early stages of anther development starting with the initiation of the archesporial cells. Megasporogenesis was also found to be affected in spl8 mutants such that some megasporocytes degenerated at an early stage (Unte et al. 2003).

To determine whether the sterility of SPL8 gain-offunction was due to defects in anther or ovule development, 35S::SPL8 transgenic plants and spl8 mutants were manually pollinated with wild-type pollen. Spl8 mutant siliques contain many aborted ovules even after pollination with fully viable wild-type pollen (Fig. 2A), confirming the previous reported abnormal megaspore development in spl8 (Unte et al. 2003). Unlike spl8 mutants, the constitutive SPL8 overexpressing plants seem not to be affected in ovule development. Their siliques showed full seed set after manual pollination with wild-type pollen (Fig. 2B).

We also compared stamen structures at stage 9 of anther development (staging according to Sanders et al. 1999) since it was shown that spl8 mutants have abnormally short filaments and small anthers (Unte et al. 2003). In sharp contrast to spl8 (Fig. 2E), stamens of $35 S:: S P L 8$ (Fig. 2D) did not differ notably from wild type (Fig. 2C). This observation suggested that anther defects in $35 S:: S P L 8$ plants start at a relatively late stage of development. Interestingly, we noticed an obvious increase of trichome density and complexity on the abaxial side of the sepals in $35 S:: S P L 8$ flowers (Fig. 2G), which is to the opposite of spl8 mutants (Unte et al. 2003; Fig. 2H).

A histological analysis was performed to determine at which stage the anther developmental defects appeared. It turned out that an inability of the anthers to dehisce caused the observed male sterility in $35 S:: S P L 8$ plants. At anther stage 11, wild-type tapetum starts to degenerate and endothecial cells enlarge (Fig. 3A, B). Anthers of $35 S:: S P L 8$ flowers show comparable endothecial enlargement and tapetal degeneration (Fig. 3E, F). The only recognizable difference is that the middle layer, originally a single cell layer between endothecium 


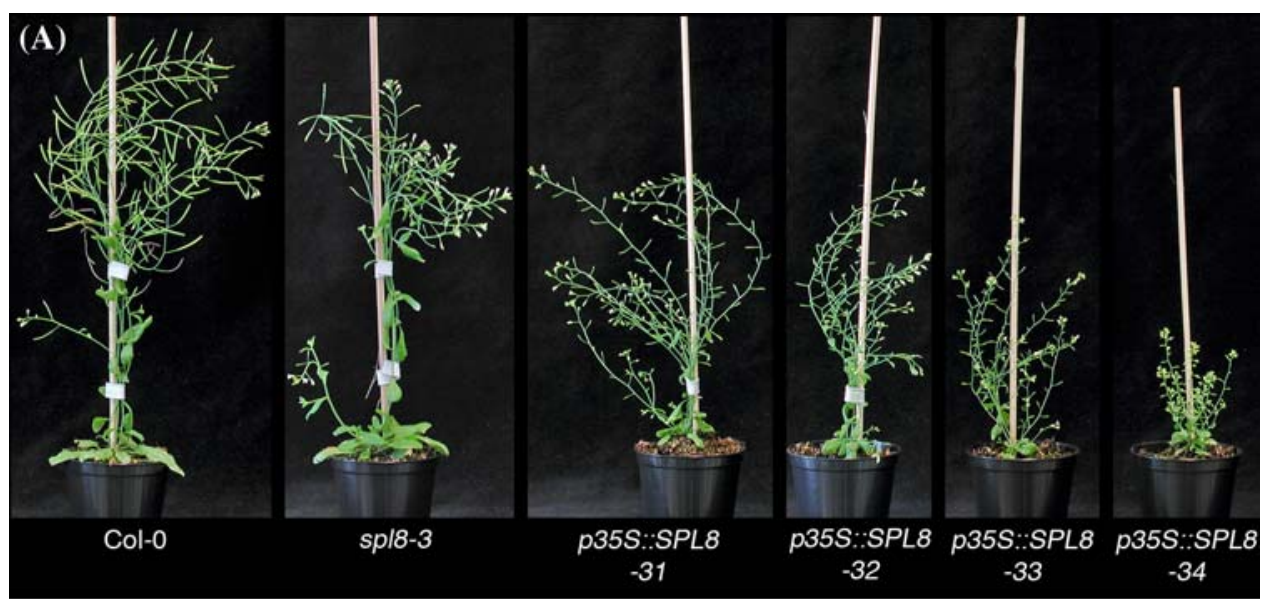

(B)

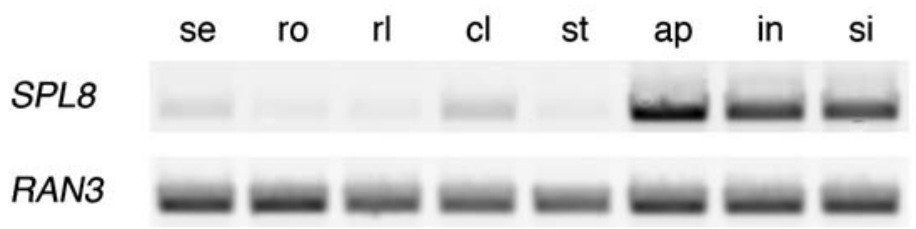

Fig. 1 The spatiotemporal expression of $S P L 8$ is important for proper plant development. (A) Independent $S P L 8$ overexpressing transgenic lines ( $p 35 S:: S P L 8-31$ to -34$)$ show a heritable and, although to a different degree, consistent pleiotropic phenotype, including sterility (note short and seedless siliques in comparison to Col-0 wild-type plant on the left). An spl8 loss-of-function

and tapetum and disappearing at stage 8 in wild-type anthers, is still present in $35 S:: S P L 8$ anthers at stage 11 (Fig. 3F).

Anther stage 13 is the stage where in wild-type dehiscence happens (Sanders et al. 1999). Anther dehiscence requires secondary wall thickening through the deposition of fibrous bands (lignification) in the connective and endothecial cells (Fig. 3C, D). Subsequent enlargement of the endothecial cells together with a degenerating septum and stomium, results in rupture of the anther wall and to a release of the mature pollen grains (Fig. 3D). However, we found deposition of fibrous bands to be largely absent in connective and endothecial cells of $35 S:: S P L 8$ anthers at a comparable stage. Instead, these cells had accumulated some granular material (Fig. 3G, H). Furthermore, older $35 S:: S P L 8$ anthers showed no sign of breakage of septum and stomium. As a result, pollen grains remained trapped inside the pollen sacs. The pollen sacs of $35 S:: S P L 8$ anthers at these later stages were found to be covered with a dark-staining layer on their inside and contained, besides pollen grains, many small cellular structures of unknown origin (Fig. $3 \mathrm{H})$. mutant (spl8-3) is shown for comparison. (B) RT-PCR analysis of SPL\& transcript levels in total RNAs isolated from different tissues and organs of wild type. RAN3 was used as a loading control. se, seedlings; ro, roots; rl, rosette leaves; cl, cauline leaves; st, stem; ap, shoot apical region; in, inflorescence; si, siliques

Anther non-dehiscence in $35 S:: S P L 8$ is jasmonate independent

Jasmonates (JAs) are known to be a major factor affecting anther dehiscence. Mutants defective in JA biosynthesis or signalling typically show delayed anther dehiscence and pollen inviability (reviewed by Scott et al. 2004). We hence tested the effect of exogenous JA on $35 S:: S P L 8$ plants. However, application of methyl jasmonate (MeJA, converts to JA) did not counteract the failure of $35 S:: S P L 8$ anthers to dehisce. A pollen viability assay also showed no obvious difference between wild-type (88.0\%) and 35S::SPL8 plants $(78.3 \%)$. Together, it is not likely that $35 S:: S P L 8$ plants are disturbed in JA biosynthesis and/or signalling.

Anther non-dehiscence in $35 S:: S P L 8$ is likely due to a constitutive GA response

GA has also been hinted as a factor affecting anther dehiscence. Studies in wheat and barley demonstrated that GA overdose and constitutive GA response cause anther non-dehiscence (Colombo and Favret 1996; Murray et al. 2003). Considering the anther defects 

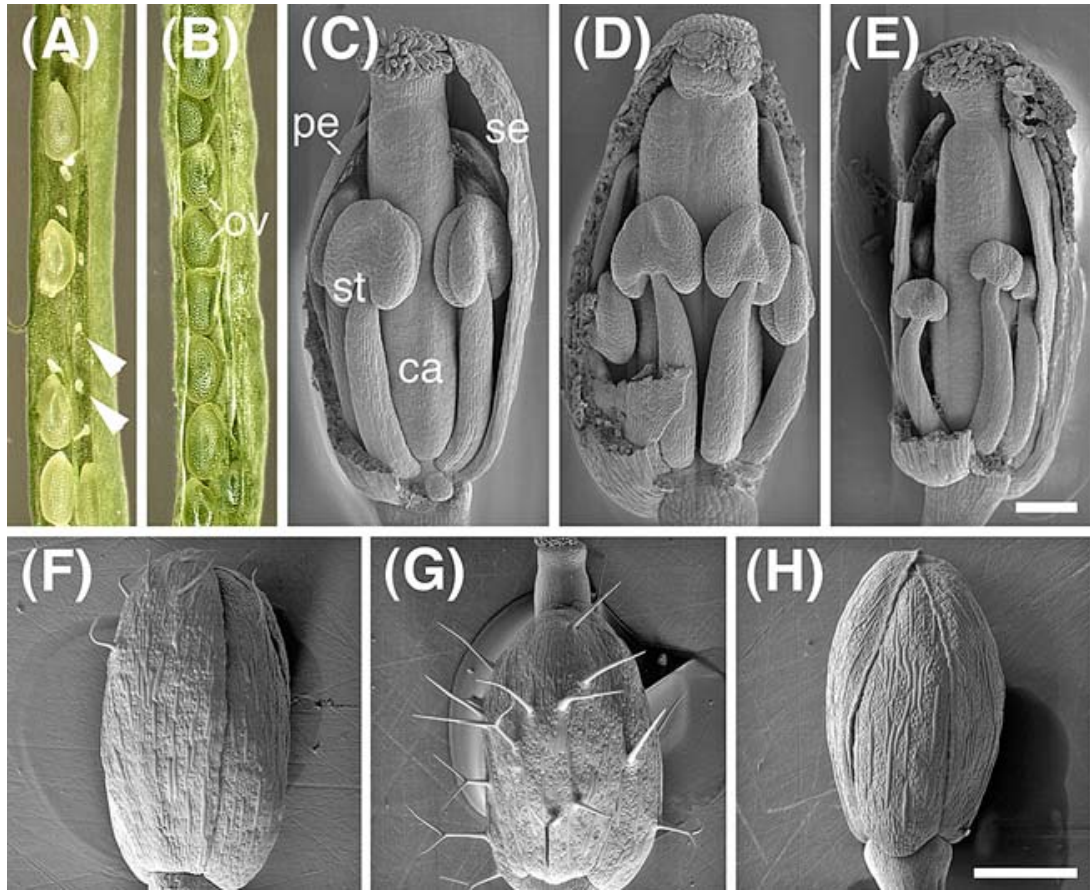

Fig. 2 Comparison of $S P L 8$ gain- and loss-of-function in reproductive growth. (A)-(B) Manual pollination could not prevent the abortion of many ovules (white arrowheads) in siliques of the spl8-1 mutant (A) but resulted in full seed set of 35S::SPL8 transgenic siliques (B). (C)-(E) SEM images of floral buds at proximately anther stage 10 after the completion of meiosis in wild-type (C), and of $35 S:: S P L 8$ transgenic (D) and

seen in SPL8 gain- and loss-of-function mutants, we reasoned that anther non-dehiscence of $35 S:: S P L 8$ could be due to a constitutive GA response.

To verify that in Arabidopsis too, a constitutive GA response leads to non-dehiscent anthers, we examined the anther histology of GA mutant spy-3 (Jacobsen and Olszewski 1993). Cross-sections made of spy-3 anthers before stage 11 were indistinguishable from those of wild type (data not shown). At stage 13 spy-3 anthers indeed showed a non-dehiscent phenotype. As mentioned before, wild-type anthers dehisce at stage 13 due to a series of events, including the enlargement of endothecial cells, deposition of fibrous bands in connective and endothecial cells, degeneration of septum and stomium (Fig. 4A, C). Spy-3 anther at a comparable stage did show the deposition of fibrous bands but to a lesser extent (Fig. 4B, D). The degeneration of septum and stomium did not happen, which might thus be the major cause for the failure to dehisce.

Seed germination and root elongation were affected by SPL8 gain- and loss-of-function

Except for anther development, GAs also play prominent roles in seed germination and root elongation spl8-1 mutant plants (E) at comparable stages. (F)-(H) Compared to wild-type (F), 35S::SPL8 transgenic sepals (G) showed an increased number of often stellate trichomes, whereas spl8-1 mutant sepals (H) almost completely lacked trichomes. ca, carpel; ov, ovule; pe, petal; se, sepal; st, stamen. Bar $=200 \mu \mathrm{m}$ in $\mathrm{C}$ to $\mathrm{E}$. Bar $=500 \mu \mathrm{m}$ in $(\mathbf{F})-(\mathbf{H})$

(Lee et al. 2002; Tyler et al. 2004; Fu and Harberd 2003).

Germination rate of $35 S:: S P L 8$ seeds was found to be $60 \%$, a strong reduction compared to that of wildtype seeds, i.e., $98 \%$, of same age and raised under identical conditions (Fig. 5A). A germination test was conducted to examine if the reduced germination potential of $35 S:: S P L 8$ seeds could be rescued by GAs. Concentrations of up to $50 \mu \mathrm{M} \mathrm{GA}_{3}$ in the medium could not, however, restore 35S::SPL8 seed germination to wild-type rates (Fig. 5A), suggesting that this phenotypic feature rather reflects GA-insensitivity than reduced endogenous GA levels.

Concerning germination potential, seeds of spl8 mutants did not differ from wild type (Fig. 5C). Interestingly, we observed that the spl8 allelic mutants showed an increased resistance to the GA biosynthetic inhibitor PAC during seed germination. Whereas the germination rate of wild-type seeds decreased from $93 \%$ to $20 \%$ in the presence of $10 \mu \mathrm{M}$ PAC, the germination rates of the spl8 mutants remained well above $50 \%$ (Fig. 5C).

Constitutive SPL8 overexpression also reduced root elongation significantly (Fig. 5B). At 9 DAG, roots of 
Fig. 3 Male sterility of constitutive SPL8 overexpression is due to nondehiscent anthers. (A) $-(\mathbf{D})$ Cross-sections through wildtype anthers at stage 11 when fibrous bands become visible in the endothecium (A) $-(\mathbf{B})$, and stage 13 when the anther ruptures along septum and stomium due to dehiscence (C) $-(\mathbf{D})$. (E) $-(\mathbf{H})$ Crosssections through $35 S:: S P L 8$ anthers at comparable stages without obvious lignification but formation of granular material in endothecial cells and still traces of middle layer visible (E)-(F). 35S::SPL8 anthers did not dehisce and pollen sacks were covered with a dark staining layer on their inside (black arrowheads) and filled with cellular structures $(\mathbf{G})-(\mathbf{H})$. co, connective; cs, cellular structures of unknown origin; en, endothecium; ep, epidermis; fb, fibrous bands; gm, granular material; ml, middle layer; pg, pollen grain; so, stomium; sp, septum; ta, tapetum; vb, vascular bundle. Bar $=100 \mu \mathrm{m}$ in $(\mathbf{A}),(\mathbf{C}),(\mathbf{E})$ and $(\mathbf{G})$. Bar $=50 \mu \mathrm{m}$ in $(\mathbf{B})$, (D), (F) and (H)

Fig. 4 Comparison of nondehiscent spy mutant anther histology to wild type. Crosssections show that the endothecium of a spy-3 mutant anther becomes less lignified. (A) and (C), Crosssections through a wild-type anther at stage 13 when the anther ruptures along septum and stomium. (B) and (D), Cross-sections through a spy3 mutant anther at a comparable stage showed less deposition of fibrous bands in the endothecium and did not rupture. $\mathrm{fb}$, fibrous bands. Bar $=100 \mu \mathrm{m}$ in (A) and (B). Bar $=50 \mu \mathrm{m}$ in $(\mathbf{C})$ and $(\mathbf{D})$
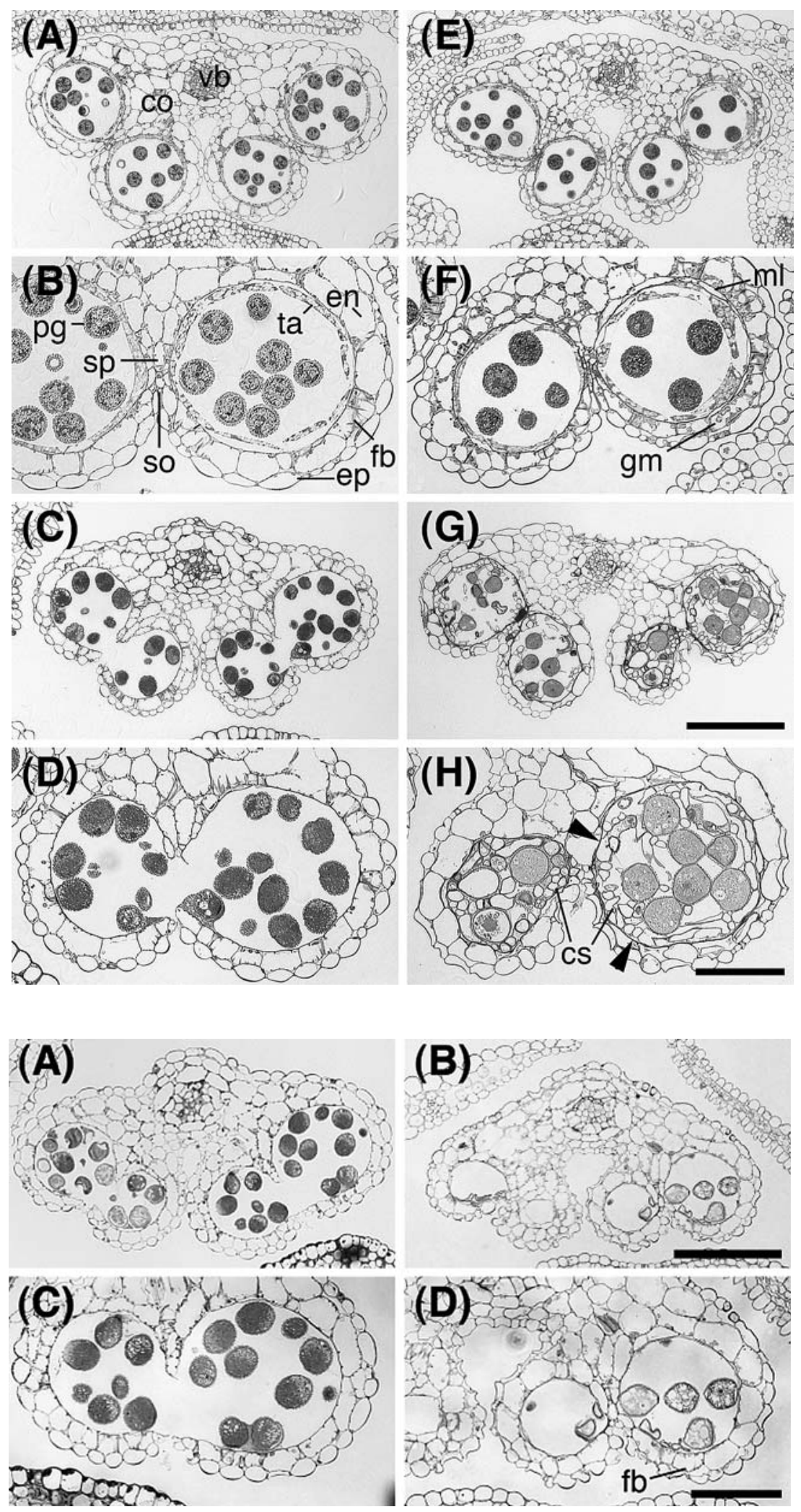

$35 S:: S P L 8$ seedlings reached only half the length of wild type, and same as the lower germination rate, extraneously applied $\mathrm{GA}_{3}$ did not affect root elongation
(Fig. 5B). In contrast, spl8 mutants revealed an enhanced root growth (Fig. 5B). Constitutive SPL8 overexpression thus conferred a certain GA-insensitivity 

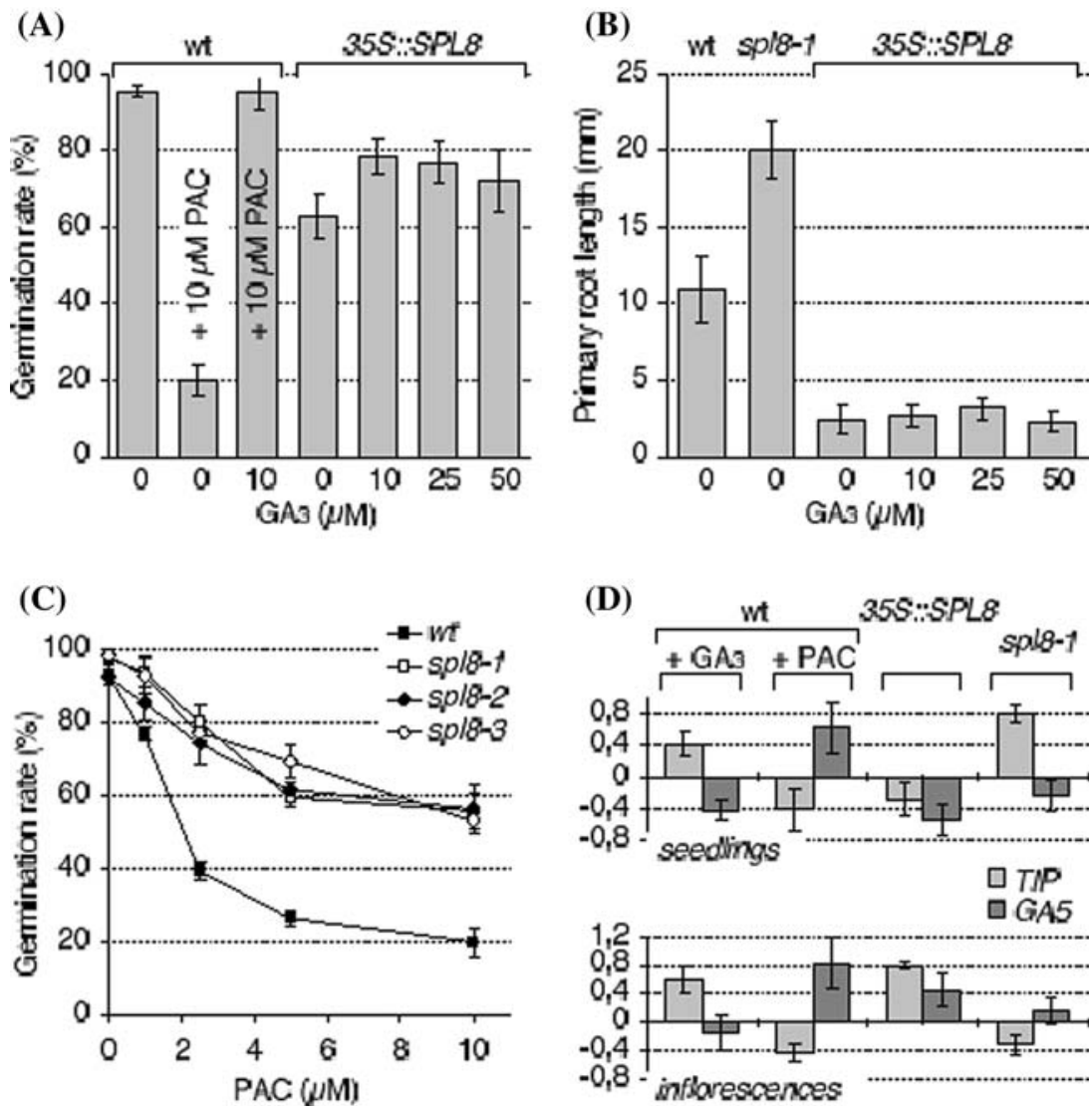

Fig. 5 Alterations in seed germination, root elongation and expression of GA biosynthesis/signalling genes in response to different SPL8 expression levels. Bioactive $\mathrm{GA}_{3}$ applied to the medium could neither rescue the low seed germination $(\mathbf{A})$ nor the reduced root length (B) of $35 S:: S P L 8$ transgenics, while spl8 mutants show an enhanced root length without GA treatment (B). (C) Germination of spl8 mutant seeds showed a higher resistance against the GA biosynthesis inhibitor PAC than wild

during the seedling stage regarding to seed germination and root elongation.

Expression of GA biosynthetic/signalling components were altered by SPL8

Alterations in endogenous GA levels/responses are generally reflected in changed transcript levels of GA biosynthetic/responsive genes. Among the typical marker genes, GA5 and $\gamma$-TIP have often been used as indicators for such alterations in Arabidopsis. GA5 encodes a key GA biosynthetic enzyme GA 20-oxidase and has found to be transcriptionally feedback controlled by GAs (Xu et al. 1999; Meier et al. 2001). $\gamma$-TIP encodes a tonoplast intrinsic protein whose transcript level is positively regulated by GAs (Cowling et al. 1998).

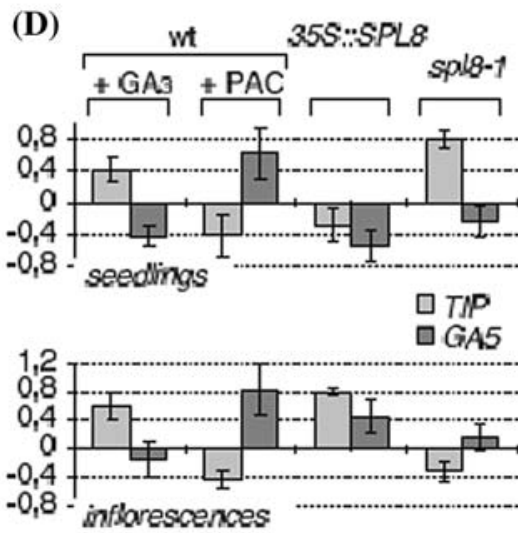

type. PAC inhibited seed germination of the wild type can be overcome by the application of $\mathrm{GA}_{3}(\mathbf{A})$. (D) GA5 and $\gamma$-TIP show altered transcript levels in seedlings (top) and inflorescences (bottom) of wild-type treated either with $50 \mu \mathrm{M} \mathrm{GA} 3$ or $10 \mu \mathrm{M}$ PAC and SPL8 gain- and loss-of-function plants. Fold changes in transcript levels are depicted in comparison to nontreated wild type after normalization against endogenous $R A N 3$ transcript levels

As SPL8 gain- and loss-of-function mutants displayed such contrasting effects in response to GA during seedling stage, we tested the possibility that $S P L 8$ affects the endogenous GA level/response. For that purpose, transcript levels of $G A 5$ and $\gamma$-TIP were compared in seedlings at $5 \mathrm{DAG}$ and in inflorescences of wild type, $35 S:: S P L 8$, and spl8-1. As shown in Fig. 5D, the transcript levels of both genes were altered by GA/PAC treatment as well as SPL8 gainand loss-of-function, indicating that SPLS indeed affects the endogenous GA level/response.

\section{Discussion}

A requirement for local acting regulators in GA signalling is inherent to the diverse local effects of GA on plant development. Most GA signalling components 
identified so far affect a full spectrum of GA regulated developmental processes, suggesting that they act rather early in the GA signalling cascade. Less is known about downstream components acting as local regulators/modulators of GA responses.

The SBP-box gene SPL8 is required for proper micro- and megasporogenesis in Arabidopsis and lossof-function mutants display a semi-sterile phenotype (Unte et al. 2003). In this report, we present evidence that SPL8 likely acts as a local GA signalling regulator/ modulator playing distinct roles depending on its spatiotemporal expression.

SPL8 positively modulates GA signalling in the flower

Sterility and reduced stamen filaments are characteristic features of most GA-deficient/insensitive mutants and depending on the plant species, GA-deficiency/ insensitivity affects different stages of anther development (Jacobsen and Olszewski 1991; Izhaki et al. 2002; Huang et al. 2003; Cheng et al. 2004). Sterility has also been reported in case of a GA overdose (Colombo and Favret 1996) and due to a constitutive GA response caused either by removing the SLENDER RICE1 gene function in rice (Ikeda et al. 2001), by removing the function of a negative GA signalling regulator $S P Y$ in Arabidopsis (Jacobsen and Olszewski 1993), by overexpressing a positive GA signalling regulator $H v G$ $A M Y B$ in barley (Murray et al. 2003) or by overexpressing a soluble GA receptor GID1 in rice (Ueguchi-Tanaka et al. 2005). Unlike GA deficiency/ insensitivity, a GA overdose/constitutive response causes anther non-dehiscence in all species studied so far (Colombo and Favret 1996; Murray et al. 2003; our study on spy-3).

In addition to abnormal stamen development, spl8 mutants showed a reduction in the density of the trichomes found on sepals, which is also known to be regulated by GA (Perazza et al. 1998). Both features could not be restored through the application of GA but spl8 mutants did respond with internode elongation in their inflorescences similar as wild type. Thus spl8 mutants have either become locally insensitive to GA or the respective floral features altered independent of GA.

Our data on SPL8 gain-of-function transgenics does support the first possibility. Plants constitutively expressing SPL8 displayed an increased sepal trichome density and complexity, normal stamen filaments and anther non-dehiscence. We ruled out two well-known possible causes of anther non-dehiscence, disturbed JA and ethylene signalling. In addition to anther nondehiscence, JA-insensitivity/deficiency results in pollen inviability, which is not the case for SPL8 gain-offunction lines. Ethylene is also unlikely to be directly related to the function of SPL8. Although ethylene deficiency/insensitivity delays anther dehiscence such that pollen release is not properly synchronized with flower opening, anther structure as such remains unaffected (Rieu et al. 2003). Based on our results, a GA constitutive response best explains the SPL8 gainof-function phenotype. That SPL8 loss- and gain-offunction resemble GA insensitive and constitutive response respectively, suggests that $S P L 8$ acts as a positive regulator in GA-mediated anther development.

SPL8 negatively modulates GA signalling in the seedling

In addition to its promoting role in reproductive growth, we found SPL8 to act as a repressor in GAmediated seed germination and root elongation. GA promotes both developmental processes through counteracting the inhibitory effect of DELLA proteins (Lee et al. 2002; Fu and Harberd 2003; Tyler et al. 2004). GA-deficiency, as observed in gal-3 mutants, results in germination failure and shortened roots (Koornneef and Van der Veen 1980; Fu and Harberd 2003). In contrast, constitutive GA-response mutants show a more PAC-resistant germination potential and can compensate for the reduced root growth of gal-3 (Jacobsen and Olszweski 1993; Lee et al. 2002; Fu and Harberd 2003; Tyler et al. 2004). With respect to seed germination and root growth, SPL8 loss- and gainof-function mutants behaved like constitutive GAresponse mutants and GA-deficient mutants, respectively.

The dual regulatory role of SPL8 in GA signalling during the seedling stage and anther development was further strengthened by molecular data. Ross (1994) hypothesized that due to an existing negative feedback mechanism, mutants with an increased GA signal transduction would have reduced levels of active GA, whereas elevated GA levels are to be expected in mutants with a decreased signal transduction. This hypothesis has found much experimental support (Cowling et al. 1998). Given that SPL8 acts as a positive modulator in GA-signalling during anther development, the enhanced GA signal transduction of $35 S:: S P L 8$ would result in reduced endogenous GA level, while spl8 mutants would have an increased endogenous GA level but reduced GA signal responses. The opposite would be expected in seedling stage considering the dual role SPL8 plays. 
$\gamma-T I P$, a gene transcriptionally activated in response to bioactive GAs (Cowling et al. 1998), was indeed shown to be up-regulated in spl8 mutants and downregulated in $35 S:: S P L 8$ transgenic seedlings in comparison to wild type. In inflorescences we observed an opposite behaviour. Based on the phenotypic and hormone treatment analysis, these data support the idea that SPL8 acts as a negative modulator in GA signalling during seedling development. It obviously performed a contrasting role during GA-regulated anther development according to our phenotypic and molecular data.

Overexpression of SPL8 resulted in elevation of $G A 5$ transcript levels in inflorescences, while lowering them in seedlings, indicating a reduced and enhanced endogenous GA level, respectively. However, there seems to be a discrepancy regarding to the $G A 5$ level found in spl8 mutants. Cowling et al. (1998) demonstrated that dominant GA-insensitive gai mutants contained high endogenous GA levels and also 4-fold higher $G A 4$ transcript levels and suggested a perturbed feedback regulation in these mutants. Similarly, removing SPL8 function could disturb a GA-dependent feedback regulation on $G A 5$.

\section{Conclusion}

In conclusion, our data let us to propose that SPL8 plays a dual role in the local regulation of a subset of GA-mediated developmental processes. SPL8 positively modulates GA signalling in the flower, whereas negatively in the seedling. Additional studies are needed to determine how SPL8 is integrated in the implementation of the GA signal. In particular, it will be interesting to find out if GA is the input signal regulating the nuclear import of SPL8 through posttranslational modification, a mechanism suggested by Birkenbihl et al. (2005).

Acknowledgements We are grateful to Susanne Höhmann for excellent technical support and Sandra Kröber for assistance in making semi-thin sections for studying anther histology. We thank Guido Jach for providing the binary vectors pGJ2148 and pGJ2171. We also thank our colleagues in the department for helpful discussions and Sabine Zachgo in particular for critically reading the manuscript. Y.Z. was supported by an International Max-Planck Research School stipend.

\section{References}

Bikenbihl RP, Jach G, Saedler H, Huijser P (2005) Functional dissection of the plant-specific SBP-domain: overlap of the DNA-binding and nuclear localization domains. J Mol Biol 352:585-596
Cardon GH, Höhmann S, Klein J, Nettesheim K, Saedler H, Huijser P (1999) Molecular characterisation of the Arabidopsis SBP-box genes. Gene 237:91-104

Cheng H, Qin L, Lee S, Fu X, Richards DE, Cao D, Luo D, Harberd NP, Peng J (2004) Gibberellin regulates Arabidopsis floral development via suppression of DELLA protein function. Development 131:1055-1064

Clough SJ, Bent AF (1998) Floral dip: a simplified method for Agrobacterium-mediated transformation of Arabidopsis thaliana. Plant J 16:735-743

Colombo N, Favret EA (1996) The effect of gibberellic acid on male fertility in bread wheat. Euphytica 91:297-303

Cowling RJ, Kamiya Y, Seto H, Harberd NP (1998) Gibberellin dose-response regulation of $G A 4$ gene transcript levels in Arabidopsis. Plant Physiol 117:1195-1203

Dill A, Thomas SG, Hu J, Steber CM, Sun TP (2004) The Arabidopsis F-box protein SLEEPY1 targets gibberellin signalling repressors for gibberellin-induced degradation. Plant Cell 16:1392-1405

Filardo FF, Swain SM (2003) SPYing on GA signalling and plant development. J Plant Growth Regul 22:163-175

Fleet CM, Sun TP (2005) A DELLAcate balance: the role of gibberellin in plant organogenesis. Curr Opin Plant Biol 8:77-85

Fridborg I, Kuusk S, Moritz T, Sundberg E (1999) The Arabidopsis dwarf mutant shi exhibits reduced gibberellin responses conferred by overexpression of a new putative zinc finger protein. Plant Cell 11:1019-1031

Fu X, Harberd NP (2003) Auxin promotes Arabidopsis root growth by modulating gibberellin response. Nature 421:740-743

Fu X, Richards DE, Fleck B, Xie D, Burton N, Harberd NP (2004) The Arabidopsis mutant sleepy $1^{\text {gar2-1 }}$ protein promotes plant growth by increasing the affinity of the SCF ${ }^{\mathrm{SLY} 1}$ E3 ubiquitin ligase for DELLA protein substrates. Plant Cell 16:1406-1418

Hay A, Kaur H, Phillips A, Hedden P, Hake S, Tsiantis M (2002) The gibberellin pathway mediates KNOTTED1-type homeobox function in plants with different body plans. Curr Biol 12:1557-1565

Hedden P, Phillips AL (2000) Gibberellin metabolism: new insights revealed by the genes. Trends Plant Sci 5:523-530

Huang S, Cerny RE, Qi Y, Bhat D, Aydt CM, Hanson DD, Malloy KP, Ness LA (2003) Transgenic studies on the involvement of cytokinin and gibberellin in male development. Plant Physiol 131:1270-1282

Ikeda A, Ueguchi-Tanaka M, Sonoda Y, Kitano H, Koshioka M, Futsuhara Y, Matsuoka M, Yamaguchi J (2001) slender rice, a constitutive gibberellin response mutant is caused by a null mutation of the SLR1 gene, an orthologue of the height-regulating gene GAI/RGA/ RHT/D8. Plant Cell 13:999-1010

Izhaki A, Borochov A, Zamski E, Weiss D (2002) Gibberellin regulates post-microsporogenesis processes in petunia anthers. Physiol Plant 115:442-447

Jacobsen SE, Olszewski NE (1991) Characterization of the arrest in anther development associated with gibberellin deficiency of the gib-1 mutant of tomato. Plant Physiol 97:409-414

Jacobsen SE, Olszewski NE (1993) Mutations at the SPINDLY locus of Arabidopsis alter gibberellin signal transduction. Plant Cell 5:887-896

Koornneef M, Van der Veen JH (1980) Induction and analysis of gibberellin-sensitive mutants in Arabidopsis thaliana (L.) heynh. Theor Appl Genet 58:257-263 
Lee S, Cheng H, King KE, Wang W, He Y, Hussain A, Lo J, Harberd NP, Peng J (2002) Gibberellin regulates Arabidopsis seed germination via $R G L 2$, a $G A I / R G A$-like gene whose expression is up-regulated following imbibition. Genes Dev 16:646-658

Magome H, Yamaguchi S, Hanada A, Kamiya Y, Oda K (2004) dwarf and delayed-flowering 1, a novel Arabidopsis mutant deficient in gibberellin biosynthesis because of overexpression of a putative AP2 transcription factor. Plant J 37:720 729

McGinnis KM, Thomas SG, Soule JD, Strader LC, Zale JM, Sun TP, Steber CM (2003) The Arabidopsis SLEEPY1 gene encodes a putative F-box subunit of an SCF E3 ubiquitin ligase. Plant Cell 15:1120-1130

Meier C, Bouquin T, Nielsen ME, Raventos D, Mattsson O, Rocher A, Schomburg F, Amasino RM, Mundy J (2001) Gibberellin response mutants identified by luciferase imaging. Plant J 25:509-519

Murray F, Kalla R, Jacobsen J, Gubler F (2003) A role for HvGAMYB in anther development. Plant J 33:481-491

Olszewski N, Sun TP, Gubler F (2002) Gibberellin Signalling: biosynthesis, catabolism, and response pathways. Plant Cell 14:S61-S80

Peng J, Carol P, Richards DE, King KE, Cowling RJ, Murphy GP, Harberd NP (1997) The Arabidopsis GAI gene defines a signalling pathway that negatively regulates gibberellin responses. Genes Dev 11:3194-3205

Perazza D, Vachon G, Herzog M (1998) Gibberellins promote trichome formation by up-regulating GLABROUS1 in Arabidopsis. Plant Physiol 117:375-383

Regan SM, Moffatt BA (1990) Cytochemical analysis of pollen development in wild-type Arabidopsis and a male-sterile mutant. Plant Cell 2:877-889

Rieu I, Wolters-Arts M, Derksen J, Mariani C, Weterings K (2003) Ethylene regulates the timing of anther dehiscence in tobacco. Planta 217:131-137
Ross JJ (1994) Recent advances in the study of gibberellin mutants. Plant Growth Regul 15:193-206

Sanders PM, Bui AQ, Weterings K, McIntire KN, Hsu YC, Lee PY, Truong MT, Beals TP, Goldberg RB (1999) Anther developmental defects in Arabidopsis thaliana male-sterile mutants. Sex Plant Reprod 11:297-322

Scott RJ, Spielman M, Dickinson HG (2004) Stamen structure and function. Plant Cell 16:S46-S60

Sorensen A, Guerineau F, Canales-Holzeis C, Dickinson HG, Scott RJ (2002) A novel extinction screen in Arabidopsis thaliana identifies mutant plants defective in early microsporangial development. Plant J 29:581-594

Steber CM, Cooney SE, McCourt P (1998) Isolation of the GAresponse mutant sly1 as a suppressor of ABI1-1 in Arabidopsis thaliana. Genetics 149:509-521

Tyler L, Thomas SG, Hu J, Dill A, Alonso JM, Ecker JR, Sun TP (2004) DELLA proteins and gibberellin-regulated seed germination and floral development in Arabidopsis. Plant Physiol 135:1008-1019

Ueguchi-Tanaka M, Ashikari M, Nakajima M, Itoh H, Katoh E, Kobayashi M, Chow T, Hsing YC, Kitano H, Yamaguchi I, Matsuoka M (2005) GIBBERELLIN INSENSITIVE $D W A R F 1$ encodes a soluble receptor for gibberellin. Nature 437:693-698

Unte US, Sorensen AM, Pesaresi P, Gandikota M, Leister D, Saedler H, Huijser P (2003) SPL8, an SBP-box gene that affects pollen sac development in Arabidopsis. Plant Cell 15:1009-1019

Xu YL, Li L, Gage DA, Zeevaart JAD (1999) Feedback regulation of GA5 expression and metabolic engineering of gibberellin levels in Arabidopsis. Plant Cell 11:927-935

Zhu QH, Ramm K, Shivakkumar R, Dennis ES, Upadhyaya NM (2004) The ANTHER INDEHISCENCE1 gene encoding a single MYB domain protein is involved in anther development in rice. Plant Physiol 135:1514-1525 